further evidence that spin transport is indeed a crucial component of ultrafast demagnetization, corroborating recent theoretical predictions $s^{5}$ and related experimental reports ${ }^{10,11}$.

The results of both studies may also be used to evaluate the magnitude of spin currents across interfaces, once all 'spin sinks' - sources of spin dissipation - are properly accounted for. The article by Choi et al. ${ }^{4}$ suggests that electron-magnon scattering is involved, but it remains unclear as to how that alone would create a spin current. More importantly, the authors are able to describe the process with the assumption that the demagnetization rate is equal to the rate of spin current generation. This confirms that demagnetization is driven by an extrinsic mechanism and that a spatial transport of spin momentum is needed, as assumed in the superdiffusive spin transport model 5 .

The observations of Schellekens et al. ${ }^{3}$ and Choi et al. ${ }^{4}$ provide clear evidence for a fast spin-transfer torque caused by a laser-induced spin current. This means that an exciting union of ultrafast magnetization dynamics and spintronics has been achieved. Their findings will surely motivate further research on how laserpumped magnetic nanostructures can drive spintronic devices at previously unthoughtof speeds.

\section{Karel Carva is at Charles University in} Prague, Faculty of Mathematics and Physics, Ke Karlovu 3, Prague 121 16, Czech Republic and in the Department of Physics and
Astronomy at Uppsala University, Box 516,

Uppsala 751 20, Sweden.

e-mail:karel.carva@mff.cuni.cz

References

1. Slonczewski, J. C. J. Magn. Magn. Mater. 159, L1 (1996).

2. Beaurepaire, E., Merle, J.-C., Daunois, A. \& Bigot, J.Y. Phys. Rev. Lett. 76, 4250-4253 (1996).

3. Schellekens, A. J., Kuiper, K. C., de Wit, R. R. J. C. \& Koopmans, B. Nature Commun. 5, 4333 (2014).

4. Choi, G.-M., Min, B.-C., Lee, K.-J. \& Cahill, D. G. Nature Commun. 5, 4334 (2014)

5. Battiato, M., Carva, K. \& Oppeneer, P. M. Phys. Rev. Lett. 105, 027203 (2010).

6. Jungwirth, T., Wunderlich, J. \& Olejník, K. Nature Mater. $11,382-390$ (2012)

Němec, P. et al. Nature Phys. 8, 411-415 (2012).

8. Bauer, G. E. W., Saitoh, E. \& van Wees, B. J. Nature Mater 11, 391-399 (2012)

9. Uchida, K. et al. Nature 455, 778-781 (2008)

10. Kampfrath, T. et al. Nature Nanotech.

8, 256-260 (2013).

11. Melnikov, A. et al. Phys. Rev. Lett. 107, 076601 (2011).

\title{
MANY-BODY PHYSICS
}

\section{At full tilt}

How does information propagate in an ordered lattice? It sounds like a simple question, but knowing when a disturbance in one corner of a system will be felt elsewhere is actually far from trivial. One possibility is that it moves at a constant speed - progressing much like the dynamics in a row of toppled dominoes (pictured). If that were the case, correlations between different parts of the system would be contained in a spatiotemporal cone, ordered manyto wonder whether perturbation in a analogous to

the causal light cones that emerge in special relativity due to the constant speed of light. When studying body systems, it's therefore natural information spreads like a row of dominoes or not.

And what better
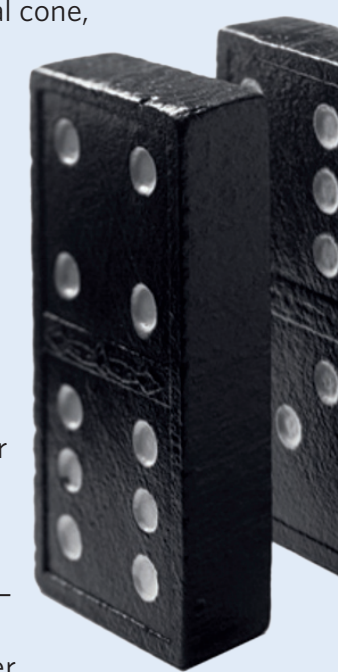
way to search for the answer than through direct simulation? That is exactly what drove two teams to independently explore the behaviour quench (Nature 511, 198-201; 2014, of a chain of ions during a global and Nature 511, 202-205; 2014). In both studies, the ion chains behaved as ensembles of spin- $1 / 2$ particles. By switching on laser-induced interactions that coupled to the collective ion motion, the groups were effectively pushing the first domino tile - all that was left to do was to watch what followed. these experiments so fascinating is that they offer the possibility of comparing both regimes, by exponent of the distance-based powerlaw scaling of the spinspin interaction.

For an approximately nearest-neighbour interaction, correlations spread between ions linearly in time - upholding the Lieb-Robinson bound. By making the range of the interaction longer and longer, the teams found that the light cone started to blur and then broke down completely. The propagation of information accelerated over time so that distant ions became correlated almost instantly.

Not only are these experiments expected to provide new insights on the effects of long-range interactions, but they also emphasize the power of quantum simulations for investigating systems that are nearly too large to be computed classically.
FEDERICO LEVI 